\title{
Interactive comment on "Southern California margin benthic foraminiferal assemblages across a modern environmental gradient record recent centennial-scale changes in oxygen minimum zone" by Hannah M. Palmer et al.
}

Anonymous Referee \#2

Received and published: 24 January 2020

This manuscript entitled "Southern California margin benthic foraminiferal assemblages across a modern environmental gradient record recent centennial-scale changes in oxygen minimum zone" by Palmer et al. presents a valuable dataset from a gradient of one of the most prominent OMZs in the world. It presents the calcareous benthic foraminiferal assemblages focusing in size fractions from core tops along a depth transect. Later the authors investigate these assemblages in short cores in order to investigate the recent history of the $\mathrm{OMZ}$ and the benthic foraminiferal assemblages. The information provided here is an important input for the ongoing investigations re- 
garding the relationship between OMZs, ecosystem and climate. Overall, I found some major details missing in this study and I believe it can be improved significantly.

1. This study is based on benthic foraminifera taxonomy work which should include references to species nomenclature also preferably a plate showing the major species mentioned. In case it is not possible to provide figures, there should be a section where list of species observed is given with references used for identification. For example: Bolivina spissa $=$ Bolivina subadvaena Cushman var. spissa Cushman 1926a. [Figures $10.7,11.4]$. This is essential for taxonomy based papers where the reader will be able to evaluate the information provided. The number of publications without any reference material is increasing and this leads the misinterpretations regarding the foraminifera research. The authors mentions their concerns in the discussion therefor I highly encourage this MS to have section dedicated to nomenclature.

2. Introduction and discussion should be improved in terms of using literature and previous work from different OMZs. For instance there are significant amount of work from the Peruvian and Arabian OMZs focusing on similar oxygen gradient and benthic foraminiferal assemblages. These studies should be included in terms of benthic foraminifera habitat in relation with oxygen and nitrate availability etc. This will improve the MS significantly. It is a pity that the species are not stained limiting the comparison with previous studies, and yet I believe the information presented here is really valuable.

3. Presentation of environmental parameters is confusing. Are these values from measurements of bottom water conditions? deepest depth of CTD? Figure 2 should be improved accordingly where stations can be shown.

4. Definition of an OMZ: please introduce OMZ already in introduction. This MS uses certain terms such as $O M Z$ edge, suboxia, hypoxic boundary and so on; to eliminate the confusion, edge or boundary of an $\mathrm{OMZ}$ considered here should be introduced as early in the text as possible.

Printer-friendly version

Discussion paper 
Notes for specific parts: page 2, line 60: please check Tetard et al., 2017.

paragraph starting with line 67: this section could be improved significantly by including previous observations from other OMZs which should be included in discussion where Bolivinids and nitrate availability are discussed.

page 3, line 107: should be "dissolved oxygen concentration"

page 4 , section under 2.3. needs to be rewritten considering the steps taken to reach the species counts. first, material sieved, dried and count in different fractions. Which references were considered for 300 and 150 specimens? Why did the authors decide these numbers? I am not an expert for statistical methods but what is the reason behind using dbRDA but not component analysis (CCA?) to test the relationship between foraminifera and environmental parameters?

line 145: "...mixed planktonic foraminifera species" please remove bulk

section 3.1.: this section is confusing. please be clear with what is presented here. I assume these are the deepest points CTD measured? is there any pore water measurements or are these only water column? and salinity should be included as well in the table.

line 205: is ANOVA introduced already in methods?

line 222: the term edge dominant.. what does this actually mean? According to which previous work edge of the $\mathrm{OMZ}$ is considered?

line 224: sentence starting with "in some taxa,.." needs rephrasing. paragraph starting from line 268: this could be written much simpler, I am not sure I understand the information provided here.

Printer-friendly version

page 9 first paragraph: we know today oxygen limited high organic matter input regions are characterised by high population low diversity of benthic foraminifera. it is interesting to see this is not the case at these sites. Nevertheless, I am not fully convinced 
the evidence provided in this study is enough to come to this conclusion. What do the authors think, if stained species were considered only the results would show any difference or not?

line 301: what does "...size fraction or 2" mean? paragraph starting at line 312: for such a discussion based on specific species, authors should provide a reference list including species names as mentioned earlier.

line 341: this is the first time specific oxygen concentration and terminology is given. This should come earlier. paragraph starting at line 365: discussion on Bolivinids: there are so many studies on bolivinids at similar setups, those should be mentioned and discussed here. Some examples are: Mallon et al., 2012; Cardich et al., (several papers), Glock et al., (several papers), Caulle et al., 2014; Jannink et al., 1998. the list goes on.

line 387: Please rephrase the last sentence. paragraph starting with line 424: it would be nice to compare results with previous observations from the region.

comment on data availability: will it be open access upon publication?

Title: I think title could be simplified and improved.

Figure 1: please give more information in the figure caption including which sites have what kind of results in the text. what are the depths of these sites?

Figure 2: water depth on y axis? station names could be implemented.

Figure 3 caption: "General observations .... " this is not needed here. Figure should be cited in the text more often.

Figure 4: y axis please mention Rel. Abundance (\%) instead.

Table 1: please add salinity and the captions should be more informative including where this information comes from. CTD? porewater? what is TOM? 
Table 2 caption: mixed planktonic foraminifera species. please remove bulk.

Interactive comment on Biogeosciences Discuss., https://doi.org/10.5194/bg-2019-446, 2019. 